\title{
Smoker's Keratosis
}

National Cancer Institute

\section{Source}

National Cancer Institute. Smoker's Keratosis. NCI Thesaurus. Code C4697.

A premalignant pathologic process that affects the oral mucosa. It is associated with the use of smoked tobacco. It appears as white lesions on the oral mucosa. Morphologically it is characterized by the pathologic production of keratin in the mucosal epithelial surface with or without epithelial atypia. It may reverse with the cessation of tobacco use. 